University of Wollongong

Research Online

Faculty of Engineering and Information

Faculty of Engineering and Information

Sciences - Papers: Part A

Sciences

$1-1-2009$

\title{
A method for reducing wave based teleoperator reflections
}

L H. Bate

University of Wollongong, Ibate@uow.edu.au

C D. Cook

University of Wollongong, chris_cook@uow.edu.au

Z Li

University of Wollongong, zli@uow.edu.au

Follow this and additional works at: https://ro.uow.edu.au/eispapers

Part of the Engineering Commons, and the Science and Technology Studies Commons

Research Online is the open access institutional repository for the University of Wollongong. For further information contact the UOW Library: research-pubs@uow.edu.au 


\title{
A method for reducing wave based teleoperator reflections
}

\author{
Abstract \\ Wave variables guarantee stability for delayed teleoperation. They also introduce reflections which can \\ prove very disorientating for an operator. This paper presents a new method for reducing the wave \\ reflections when operating in nonlinear and unknown environments. Simulations and laboratory \\ experiments shows the new method does not only guarantee stability but also reduce wave reflections \\ allowing for improved velocity tracking and force feedback.

\section{Keywords} \\ reflections, method, reducing, teleoperator, wave \\ Disciplines \\ Engineering | Science and Technology Studies

\section{Publication Details} \\ Bate, L. H., Cook, C. D. \& Li, Z. 2009, 'A method for reducing wave based teleoperator reflections', ICIT \\ 2009: IEEE International Conference on Industrial Technology, 2009, IEEE, pp. 1-6.
}




\title{
A Method for Reducing Wave Based Teleoperator Reflections
}

\author{
L.H Bate ${ }^{1}$, C.D. Cook ${ }^{2}$ and Z. $\mathrm{Li}^{1}$ \\ ${ }^{1}$ School of Electrical, Computer and Telecommunications Engineering, \\ ${ }^{2}$ Faculty of Engineering \\ University of Wollongong, Northfields Avenue, Wollongong, Australia 2522 \\ \{lhb01, c.cook, zli\}@uow.edu.au
}

\begin{abstract}
Wave variables guarantee stability for delayed teleoperation. They also introduce reflections which can prove very disorientating for an operator. This paper presents a new method for reducing the wave reflections when operating in nonlinear and unknown environments. Simulations and laboratory experiments shows the new method does not only guarantee stability but also reduce wave reflections allowing for improved velocity tracking and force feedback.
\end{abstract}

\section{INTRODUCTION}

Wave based teleoperator systems by their inherent design introduce wave based reflections. As shown in Fig. 1 and Fig. 2 they couple the velocity and force components as one which results in circulating reflections unless junction impedances are perfectly matched. These reflections can be very disorientating for the operator.

$$
\begin{aligned}
& u_{m}=\frac{b \dot{X}_{m}+F_{m}}{\sqrt{2 b}} \\
& v_{s}=\frac{F_{s}-b \dot{X}_{s}}{\sqrt{2 b}}
\end{aligned}
$$

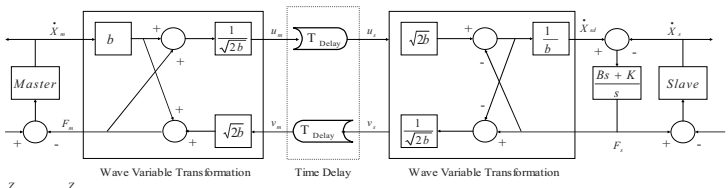

$\overrightarrow{\text { Fig. }} . \overrightarrow{1 .}$ Basic wave based Bilateral Teleoperator Impedance Junctions

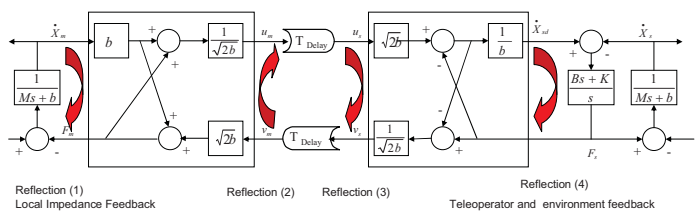

Fig. 2. Paths for Wave Reflections due to Impedance Junctions
The phenomena of reflections in Wave based Teleoperation was observed early on by Niemeyer and Slotine $[4,5]$. Their approach to reduce the wave reflections is to match the master and slave impedance using damping elements as shown in Fig. 3.

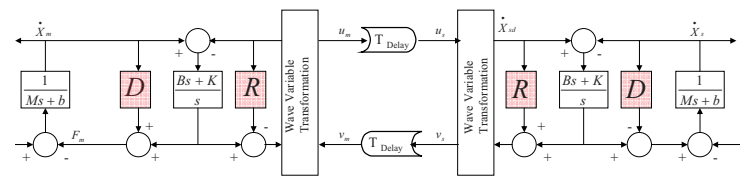

Fig. 3. Additional damping elements on both Master and Slave sides

The predetermined damping elements do not have the ability to handle unpredictable impedance changes of either operator or environment. The system also suffers from the fact that wave reflections can be reinstated when the operating environment undergoes unknown changes.

In view of this, Niemeyer devised a method to overcome unmodeled impedance changes by restricting the bandwidth of the Teleoperator by utilising wave filters [5]. The wave filter has a cut off frequency that is equivalent to that of half the lowest expected communications delay. By combining impedance matching to minimize the reflections in free space, and wave filtering to minimize reflections brought about by the operator and slave environment during the systems operation, wave reflections are significantly reduced.

However this approach to minimize wave reflections has the trade off of restricting the Teleoperator bandwidth. Additionally this predetermined filter value requires accurate estimation of the teleoperator's telecommunications delay.

Considering the required trade offs of Teleoperator system performance Hirche $[6,7]$ proposed an approach that uses a position / force Teleoperator system together with frequency domain loop shaping filters as shown in Fig. 4. Through the use of an optimization algorithm the impedance was able to be closely matched for a constant delay. The passivity of the Teleoperator system was ensured by the filter "Passivity Reserve" margin. 


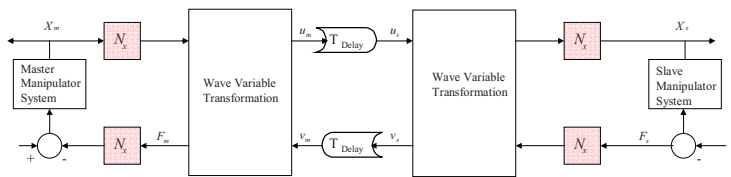

Fig. 4. Basic wave based Bilateral Teleoperator with Loop Shaping Filters

Although effective in impedance matching of the Teleoperator system, this approach also has limitations as follows:

- Impedance matching is optimized for one environment at a time

- Impedance matching requires prior knowledge of the environment and the loop shaping of the estimated environment takes considerable computation time.

Instead of $Z=$ Force/Velocity the function used to calculate the filters before operation is an analytical one of $Z=$ Force/Position and therefore the method is not able to entirely take into account the real world physical variances from the ideal eg. friction, or a change from free moving to solid contact operation.

Kuschel [8] extended the work of Hirche in order to overcome the system limitation of requiring prior knowledge of the environment, by switching between a variety of filters to enable the Teleoperator system to operate across a range of expected environments. A time variant filter is utilized to allow smooth switching between filters. To further smooth the switching, Kuschel utilized a decoupling algorithm. However to enable calculation of the filter bank range, prior knowledge of the Teleoperator systems remote environment is still required.

Munir [9] implemented a wave teleoperator with prediction. This architecture provided impressive results for free space teleoperator movement provided the teleoperator slave and the communications delay are modeled accurately. The system suffers from the fact that wave reflections can be reinstated when the operating environment undergoes unknown changes.

Thus we can observe that despite the effectiveness of all the above systems in attenuating wave reflections they all require prior estimation of either the teleoperator's remote environment or the communications delay.

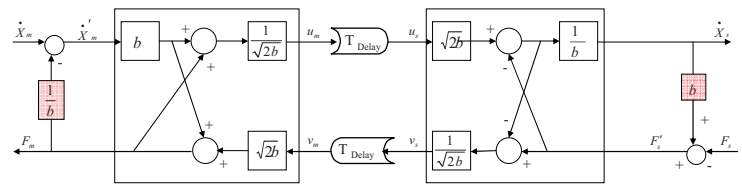

Fig. 5. Impedance Matched Teleoperator

It is also interesting to note that when a teleoperator system is perfectly matched (Fig. 5) the $u_{m}$ wave variable contains only velocity information and the $\mathrm{v}_{\mathrm{s}}$ wave variable contains only force information. Thus the wave variables become

$$
\begin{aligned}
& u_{m}=\frac{\dot{b X_{m}}}{\sqrt{2 b}} \\
& v_{s}=\frac{F_{s}}{\sqrt{2 b}} .
\end{aligned}
$$

With the perfectly impedance matched wave teleoperator the output of the wave variables $u_{m}$ and $v_{s}$ no longer contain components of the incoming wave variables $\mathrm{u}_{\mathrm{s}}$ and $\mathrm{v}_{\mathrm{m}}$. Therefore the circulating wave reflections illustrated within Fig. 2 are prevented. However the additional impedance matching terms corrupt the values of slave velocity and master force as follows.

$$
\begin{aligned}
& \dot{X}_{s}=\frac{\dot{X}_{m}}{2}(t-T)+\frac{F_{s}}{2 b} \\
& F_{m}=\frac{b \dot{X}_{m}}{2}+\frac{F_{s}}{2}(t-T)
\end{aligned}
$$

In an effort to circumvent the above problems we propose a direct active method of impedance matching which does not require a prior knowledge of the slave's operating environment and does not introduce additional delays into the Teleoperator system. It will be shown that this proposed method provides a significant improvement when the slave is operating in nonlinear environments. The remainder of this paper is organized as follows. Section 2 presents the new method. Section 3 describes simulation examples. Section 4 demonstrates experimental results and Section 5 concludes this paper.

\section{CANCELlation Within WaVe Domain}

\section{A. New Teleoperator Architecture}

The new approach (Fig. 6) alters the wave variables architecture as follows

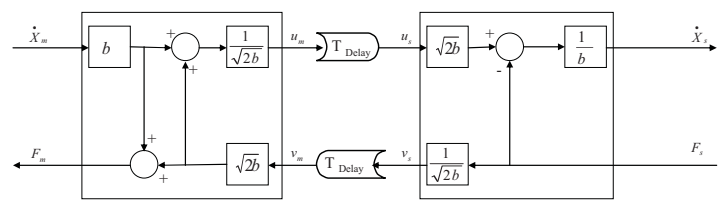

Fig. 6. Implementation of new wave-based Teleoperator

$$
\begin{aligned}
& u_{m}=\frac{b \dot{X}_{m}+F_{m}}{\sqrt{2 b}} \\
& v_{s}=\frac{F_{s}}{\sqrt{2 b}}
\end{aligned}
$$


Observing the new $\mathrm{v}_{\mathrm{s}}$ wave variable we can see that it no longer contains any velocity information; therefore the slave wave variable no longer includes the incoming wave variable. That is, the wave variable $u_{s}$ is no longer reflected at the $v_{s}$ port.

With the force and velocity decoupled at the slave side, circulating wave reflections are prevented.

The observed values of slave velocity and master force show that this new system facilitates improved velocity tracking:

$$
\begin{aligned}
& \dot{X}_{s}=\dot{X}_{m}(t-T)+\frac{F_{s}}{b}(t-2 T)-\frac{F_{s}}{b} \\
& F_{m}=b \dot{X}_{m}+F_{s}(t-T)
\end{aligned}
$$

The advantages of this new teleoperator architecture are:

1) improved velocity tracking in comparison with the impedance matched teleoperator. This can be analytically observed by comparing the Master to Slave velocity equation of the perfectly impedance matched teleoperator equation (5) with that of the new teleoperator architecture, equation (9)

2) reflection cancellation does not require the use of active modeling or impedance matching $[6,7,8,9]$ to attenuate the wave reflections.

3) no prior knowledge of the remote environment is required.

\section{B. Stability Considerations}

The wave variable controller ensure stability of teleoperator systems in the presence of undefined communication delays. This is achieved via the following passivity criteria.

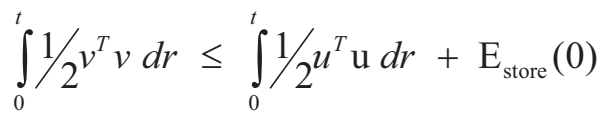

Anderson and Spong [1] state that a system is passive if and only if the norm of its scattering operator is equal to or less than one. The scattering matrix $\mathrm{H}(\mathrm{s})$ for the two port teleoperator system is as follows:

$$
\left[\begin{array}{l}
F_{m}(s) \\
-\dot{X}_{s}(s)
\end{array}\right]=H(s)\left[\begin{array}{l}
\dot{X}_{m}(s) \\
F_{s}(s)
\end{array}\right]
$$

The scattering matrix of the above two port teleoperator system is related to the hybrid matrix $\mathrm{H}(\mathrm{s})$ as follows

$$
S(s)=\left[\begin{array}{rr}
1 & 0 \\
0 & -1
\end{array}\right][H(s)-I][H(s)+I]^{-1}
$$

The new wave based teleoperator system is as follows:

$$
\left[\begin{array}{c}
F_{m} \\
\dot{-X}_{s}
\end{array}\right]=\left[\begin{array}{ll}
b & e^{-s T} \\
-e^{-s T} & \frac{e^{s T}-e^{-s T}}{b e^{s T}}
\end{array}\right]\left[\begin{array}{l}
\dot{X}_{m} \\
F_{s}
\end{array}\right]
$$

$$
H(s)=\left[\begin{array}{ll}
b & e^{-s T} \\
-e^{-s T} & \frac{e^{s T}-e^{-s T}}{b e^{s T}}
\end{array}\right]
$$

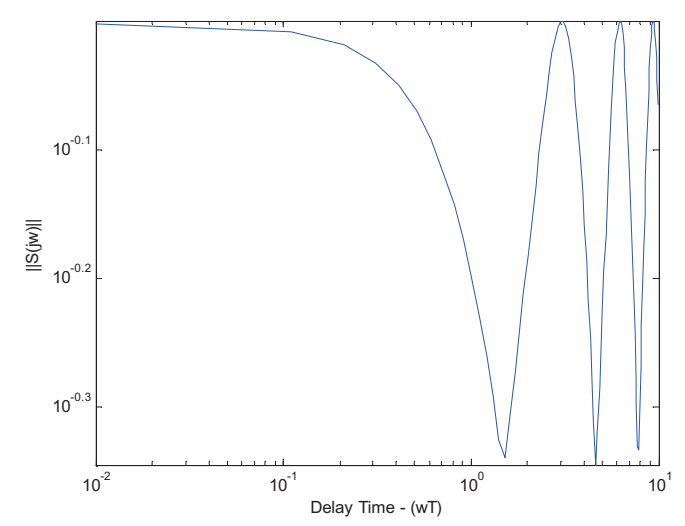

Fig. 7. Scattering matrix norm of new teleoperator architecture.

The hybrid matrix produces a scattering norm of $\|S(s)\| \leq 1$ thus satisfying the passivity criteria and therefore the new teleoperator architecture can be considered stable. Fig. 7 confirms by graphical representation that the scattering norm is less than or equal to one for the given communications delay range of between 0.01 seconds to 10 seconds

\section{Simulation Results}

This section compares the performance of the original telecommunication system with the newly developed teleoperation system. A very demanding situation in which the same teleoperator is expected to operate in free space, and then move into a solid wall, was chosen to test the new theory.

The time delay for these simulations is set to a fixed $200 \mathrm{mS}$ ( $400 \mathrm{mS}$ round trip time); this is comparable to the round trip transit time of an Internet User Datagram Packet (UDP) from Australia to the USA [10]. For all simulations 
the master manipulator is moved forward and is kept there for approximately 6 seconds. Following this the master manipulator is moved back to the initial home position.

In Fig. 8 the teleoperator is moving in free space. The problematic wave reflections are easily observable both in the Slave position oscillation and the circulating force reflections. The human operator (the master) would find the circulating force reflections very disorientating.
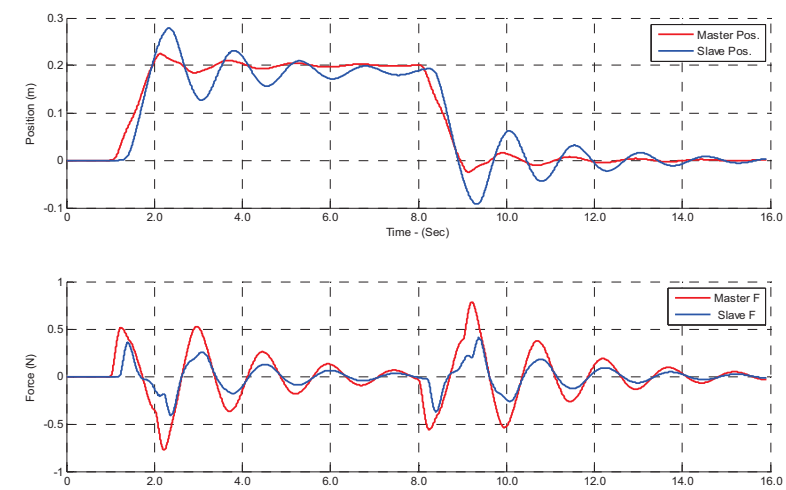

Fig. 8. Original wave-based teleoperator - Free Space
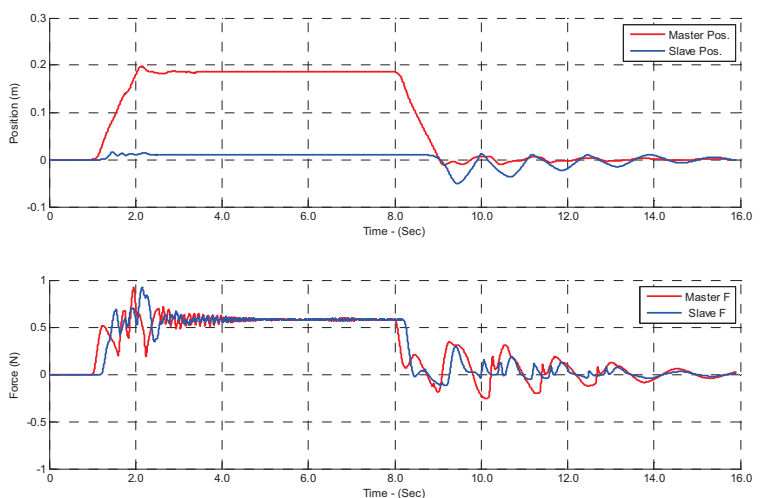

Fig. 9. Original wave-based teleoperator - Wall

In Fig. 9 the teleoperator slave hits a simulated wall located $1 \mathrm{~cm}$ from the initial slave position. Again the disorientating effects of the circulating wave reflections can be observed both in the position and force domains.
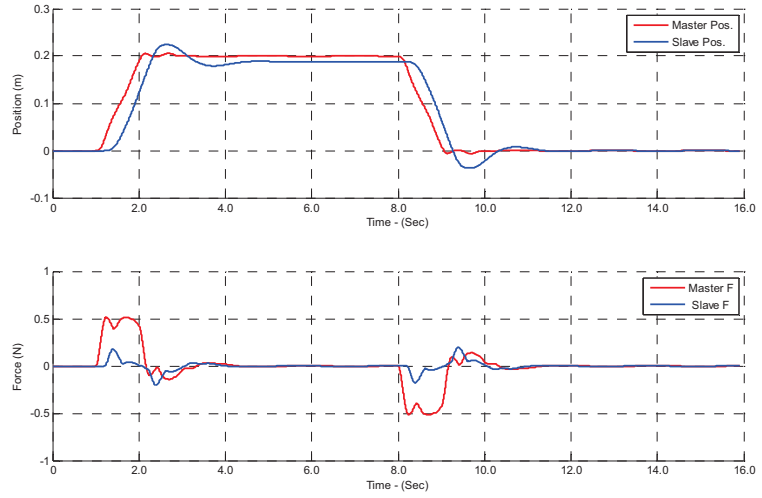

Fig. 10. New wave-based teleoperator architecture - Free Space
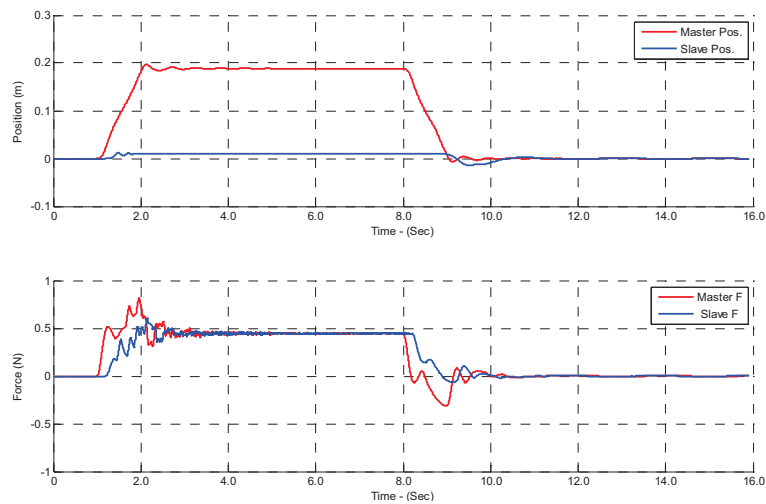

Fig. 11. New wave-based teleoperator architecture - Wall

Fig. 10 and Fig. 11 illustrate the performance of the new wave based teleoperator system operating under the same conditions. The improved transient response of the new teleoperator architecture can be clearly observed; this is due to the significant attenuation of the circulating wave reflections.

Essentially the previous disorientating Slave position and force oscillations are almost completely removed thus providing the operator of the teleoperator a far closer representation of the remote Slave environment even when the environment changes drastically from free space to hitting a solid object.

\section{EXPERIMENTAL RESULTS}

In this section, we illustrate the operation of the new wavebased teleoperator architecture on a real world test bed utilizing the Mathworks xPC toolbox and AC servo motors to represent Master and Slave manipulators.

The test bed setup consists of one AC servo motor with a $0.3 \mathrm{~m}$ rod directly coupled to the shaft to represent a single degree of freedom Master manipulator. The second AC motor is configured is the same manner to represent the Slave 
manipulator. The kinematically similar Master and Slave manipulators are controlled by individual AC servo drives.

Each AC servo drive is controlled in real-time by the Mathworks xPC real-time target PC. The control loop is configured at a $1 \mathrm{KHz}$. Sample rate.

The communications time delay for the test bed is set to the same fixed $200 \mathrm{mS}$ ( $400 \mathrm{mS}$ round trip time) as that of the previous simulations.
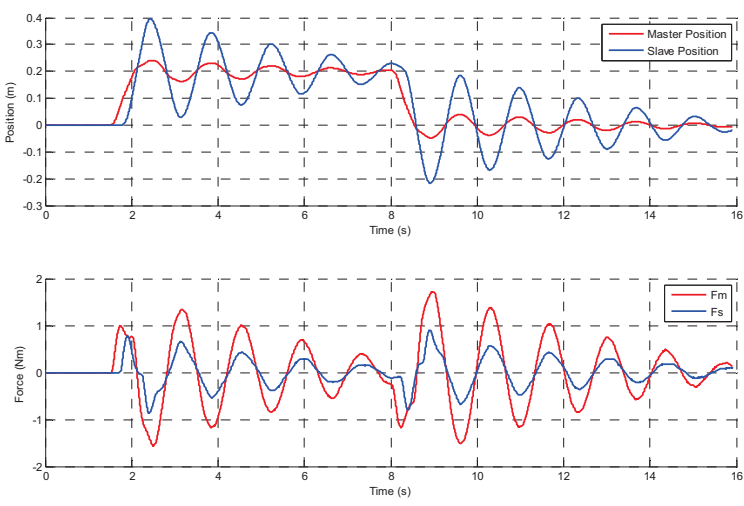

Fig. 12. Original wave-based teleoperator - Free space
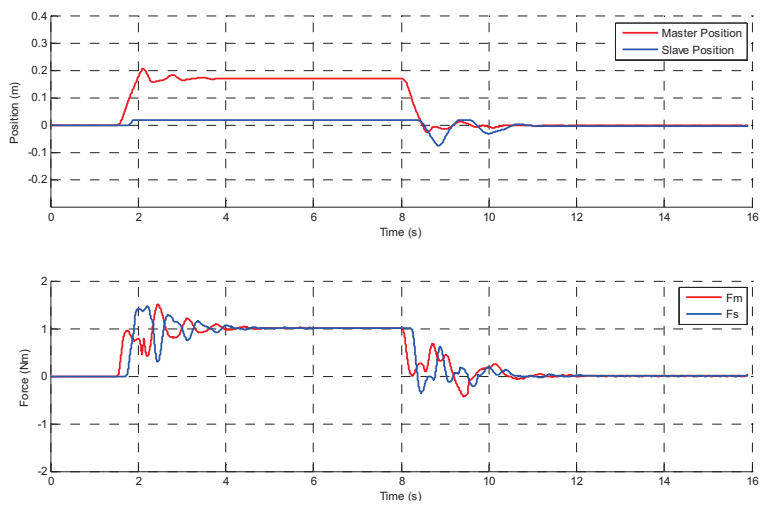

Fig. 13. Original wave-based teleoperator - Wall

In Fig. 12 the original wave based teleoperator architecture as outlined in Fig. 1 is moving in free space. The problematic wave reflections are easily observable both in the Slave position oscillation and the circulating force reflections.

In Fig. 13 the teleoperator slave contacts a solid wall located hits a simulated wall located $2 \mathrm{~cm}$ from the initial slave position.

The performance improvement of the new wave based teleoperator system (Fig. 6) when operating under the same conditions to that of the original wave based teleoperator design can be directly compared by to that of the original wave based teleoperator in following results of Fig. 14 and Fig. 15.

Firstly in Fig. 14 where the new teleoperator architecture is moving in free space the improved transient response is clearly observable when compared to that of the original wave based teleoperator in Fig. 12.
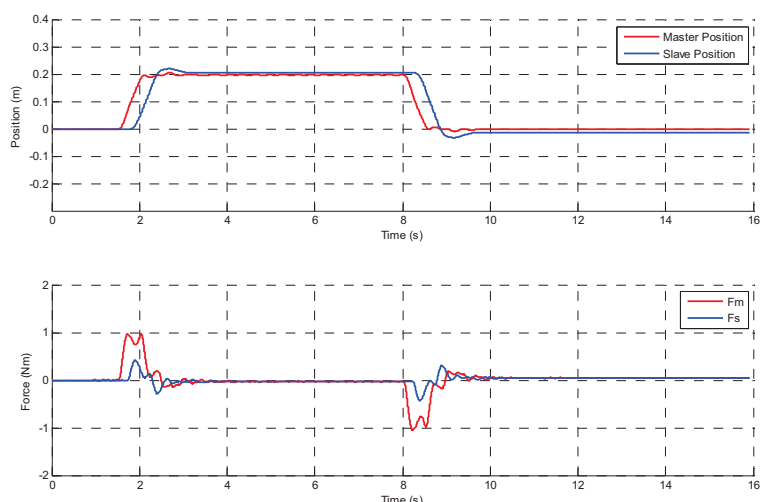

Fig. 14. New wave-based teleoperator architecture - Free space
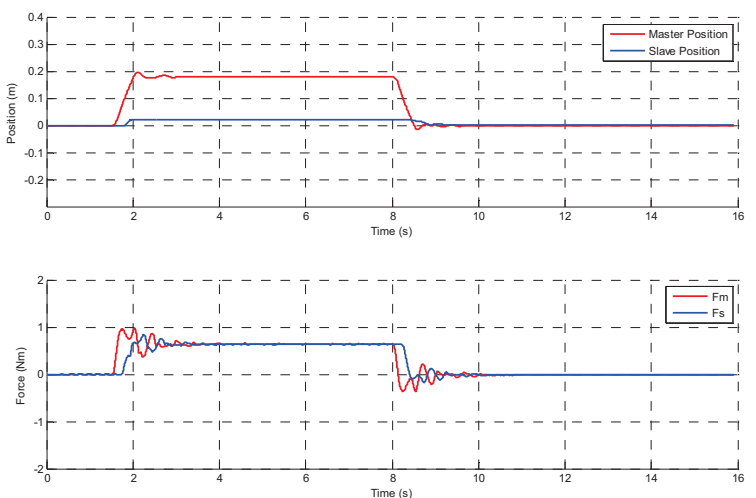

Fig. 15. New wave-based teleoperator architecture - Wall

Fig. 15 confirms the new teleoperator architecture performance improvement to that of the original teleoperator architecture operating in the identical environment (Fig. 13) is attained for the challenging environment of the transition from free space to hitting a solid wall.

\section{CONCLUSIONS}

This paper presents a new wave teleoperator architecture which significantly reduces wave based reflections while maintaining stability.

This new method negates any requirement to prior modeling of the remote environment to facilitate the use of active tuning / compensation to avoid wave based reflections. This enables the system to have superior performance when operating within complex nonlinear environments compared with teleoperator architectures that rely on active tuning through modeling of the remote environment.

Simulation and experimental results were presented which showed teleoperation able to cope well with an environment which changed from free space to a solid object. These results 
confirmed the significant attenuation of the wave based reflections, and showed how this can facilitate improvement to the operator's perception of the remote slave manipulators environment.

\section{REFERENCES}

[1] R. Anderson and M. Spong, "Bilateral Control of teleoperators with time delay", Decision and Control, Proceedings of the 27th IEEE Conference, 7-9 Dec. 1988, pp. $167-173$

[2] G. Niemeyer and J. Slotine, "Stable adaptive teleoperation", IEEE Journal of Oceanic Engineering, Vol 16 No.1, pp. 152-162, 1991

[3] D Lawrence, "Stability and transparency in bilateral teleoperation, Proceedings of the 31st IEEE Conference, Decision and Control, Tucson Arizona, 1992, Dec. 1992, pp. 2649 - 2655

[4] G. Niemeyer and J. Slotine. Transient shaping in force-reflecting teleoperation, Proceedings of the Int. Conf. on Advanced Robotics, volume 1, pp. 261-266, June 1991

[5] G. Niemeyer, "Using wave variables in time delayed force reflecting teleoperation" Ph.D. Thesis, Massachusetts Institute of Technology, Cambridge, MA, September 1996

[6] S. Hirche and M. Buss, "Study of teleoperation using realtime communication network emulation", Proceedings of IEEE/ASME International Conference on Advanced Intelligent Mechatronics AIM'03, (Kobe,Japan), 2003, pp. 586-591

[7] S. Hirche and M. Buss, "Passive position controlled telepresence systems with time delay", Proceedings of the American Contro Conference ACC'03, (Denver, US), 2003, pp.168-173.

[8] M. Kuschel, "Performance oriented control of haptic telepresence systems", Thesis Munich, October 1, 2004, (Thesis Adviser Sandra Hirche)

[9] Saghir Munir. "Internet-based teleoperation", $\mathrm{PhD}$ Thesis, Georgia Institute of Technology, 2001

[10] L. Bate, C. Cook, "Exploration of Intelligible Force Feedback for TeleSurgery over the Internet", International Conference on Information Technology in Mechatronics, 1-6 Oct 2001, pp. 124 - 129 